\title{
Dry powder inhalation of siRNA
}

\author{
Tobias WM Keil ${ }^{1}$ \& Olivia M Merkel*,1 (iD) \\ ${ }^{1}$ Pharmaceutical Technology \& Biopharmaceutics, LMU München, Butenandtstr. 5-13, 81377 München, Germany \\ *Author for correspondence: Tel.: +49 892180 77025; Olivia.Merkel@Imu.de
"dry powder formulations are a promising dosage form to locally deliver target-specific siRNA enabling the therapy of several diseases such as asthma, cystic fibrosis or lung cancer. "

First draft submitted: 20 March 2019; Accepted for publication: 1 April 2019; Published online: 16 May 2019

Keywords: dry powder inhaler • inhalation • nanoparticle • siRNA • spray drying • spray freeze drying

\section{siRNA-based nanoparticles}

Therapeutic RNA interference (RNAi) recently gathered a new wave of public interest due to the approval of the first small interfering RNA (siRNA)-based drug (patisiran) against hereditary transthyretin amyloidosis [1]. Exogenous double-stranded RNA can be used to trigger this interference by an intracellular complex known as RNA-induced silencing complex after 'dicing' the molecule to 21-23 nucleotides. Depending on the base pairing of this siRNA, the complex attaches to a specific messenger RNA (mRNA) and ultimately degrades it. Thus, a subsequent translation to the protein encoded by this particular mRNA is prevented. By choosing the siRNA sequence for subsequent base pairing, any possible mRNA/protein of interest can be downregulated, including mRNAs that cause a disease.

The uptake of siRNA into the cytoplasm, however, is restricted by its chemical nature. High hydrophilicity and large size of siRNA compared with small molecules lead to a low uptake. To overcome these problems, vectors have been developed based on viral and nonviral delivery systems. Viral delivery systems, such as adenoviruses, adenovirus-associated viruses and lentiviruses, which are the most frequently tested viruses in literature, are able to successfully deliver siRNA. However, these vectors have great disadvantages such as high immunogenicity, high production costs and major hurdles for regulatory approval [2]. Nonviral systems, such as lipids or polymers, show lower immunogenicity, longer circulation times and lower costs, but also yield lower uptake efficiency. Nonetheless, when targeting inflammatory diseases in which additional immune response is counterproductive, these systems are favored and their shortcoming is addressed, for example by adjusting the administration route. In addition, nonviral systems can be modified due to their synthetic nature to optimize biocompatibility, internalization, transfection efficiency, circulation time and active targeting and improve their overall performance [2,3]. Lipid and polymer delivery systems can either form vesicle-based nanoparticulate suspensions upon encapsulation or lipoplexes and polyplexes, respectively, upon complexation with charged substances [2,4]. A benefit of a nanoparticle formation is that siRNA is protected from RNases and, hence, is less susceptible to enzymatic degradation. RNases, which are ubiquitously present in eukaryotes, prokaryotes, bodily fluids and generally in every source of water, are one of the major risks in siRNA formulations. Therefore, it is of great interest to avoid sources of contamination and also the presence of water without which RNases lose their enzymatic activity. Hence, storage of siRNA-based medicines is best in solid formulations.

\section{Dry powder inhalation}

Pulmonary delivery is an optimal way to treat lung diseases such as asthma, chronic obstructive pulmonary disease, cystic fibrosis and lung cancer, avoiding the oral and intravenous route. Local administration onto the lung area is complemented by reduced doses, subsequently reduced systemic side effects, and circumvents the first past metabolism [5].

For pulmonary delivery, drugs are delivered to the lung via liquid aerosol or dry powder formulations. Liquid aerosols generated by pressurized metered dose inhalers are mostly favored for small molecule delivery as they are 
easy to formulate. However, administration of pressurized metered dose inhalers needs some training for the patient to apply the medication correctly achieving best possible results. In detail, the timing of inhaling and the aerosol release needs to be coordinated and has to be done simultaneously. Otherwise, drug deposition will take place in the mouth and pharynx and not at the site of action within the lung, resulting in potential side effects [6]. On the other hand, dry powder inhalers (DPIs) release their drug formulation upon the patient's respiration. Hereby, coordination of breathing and drug release is not required, easing the handling of the device and increasing patients compliance [7]. Upon inhalation of the patient, the dry powder formulation is simultaneously released. Through the generated vacuum and the device's specific structure, possible agglomerates of particles are disaggregated [8]. However, these DPIs need certain respiration forces to trigger the release mechanism. In some cases, where airflow and, hence, respiration force is restricted (e.g., by the disease itself or the patient's anatomy [children]) it is not strong enough to release the DPI's content. To overcome these problems, various DPIs were approved with different release mechanisms to fully adapt DPIs to the patient's needs and therefore enable applicability $[2,7,9,10]$. Advantages of DPIs over MDIs can be seen when water-sensitive drugs are formulated, such as siRNA-related medicines. In the absence of water, degradation caused by enzymatic and nonenzymatic reactions cannot take place [2]. For this purpose, dry powder formulations need to be produced with low residual moisture content, and the formulation itself needs to be protected from environmental humidity. For capsule-driven DPIs, which are the most applied systems in early research stages due to easy filling and handling, typically a higher water resistive material is chosen for capsule production, that is instead of gelatin with about $10 \%$ residual moisture content, the most common capsule material, hydroxypropylmethylcellulose is used. This substance exhibits a residual moisture content of less than $1 \%$ and owns additional beneficial properties, for example, less powder adhesion to the inner wall of the capsule, compared with gelatin [7,11]. In addition, primary packaging materials such as aluminum films significantly restrict the diffusion of air and humidity through the packaging material into the dosage form preventing wetting of, for example, capsules and their powder content.

\section{Microparticle preparation techniques}

Techniques that are frequently reported in literature to produce dry powder formulations are spray drying (SD) and spray freeze drying (SFD) [12-15]. Hereby, siRNA nanoparticles are suspended in an aqueous excipient solution (e.g., mannitol or trehalose) and afterward aerolized into small droplets and dried resulting in microparticles in which these nanoparticles are embedded. This procedure is necessary as particles between 1 and $1000 \mathrm{~nm}$ are not able to deposit in deep lung areas because they are exhaled upon inhalation. Suitable aerodynamic sizes for lung deposition, which depend on geometric size and porosity, are between 1 and $5 \mu \mathrm{m}$ [16]. Hence, nanoparticles need to be embedded into a microparticle which dissolves upon impaction on the lung fluid and releases its nanoparticle content. When using SD, microparticles are produced via gentle heating, whereas SFD techniques utilize the freezing of droplets in liquid nitrogen at first and subsequent removal of frozen water by lyophilization. Both preparation methods produce mostly microparticles in an amorphous state, which is favorable for stabilizing embedded nanoparticles. Whereas SD results in rather small geometric sizes with low porosity, SFD produces very voluminous particles with high porosity. These high volumes might cause powder packaging and/or application issues for SFD microparticles. On the other hand, higher temperatures necessary for SD are avoided [17]. Despite the different geometric sizes, both techniques are able to produce microparticles with an aerodynamic diameter between 1 and $5 \mu \mathrm{m}$, due to their different particle porosities. Beneficial for industrial particle production by SD is the short time of drying which only takes minutes compared with several hours or days for SFD. Great advantage of both techniques is the ability to tune and design the microparticle by adjusting process parameters of the respective techniques: pump rate, airflow and solid concentration (for SD also aspiration rate and heat apply here) have a crucial effect on particle size and shape $[18,19]$. In addition, microparticle morphology can be modified by excipients and further additives. Leucine, for example, is known to roughen the particle surface avoiding strong particle-particle interaction and improving aerodynamic properties [20].

As mentioned before, siRNA-based nanoparticles embedded in microparticles demonstrate increased siRNA stability due to the absence of water, which avoids enzymatic degradation through RNases.

\section{Conclusion}

In summary, dry powder formulations are a promising dosage form to locally deliver target-specific siRNA enabling the therapy of several diseases such as asthma, cystic fibrosis or lung cancer. In literature, siRNA-containing 
microparticles produced by SFD successfully demonstrated gene silencing activity in vivo [15]. Nevertheless, further research is required to realize this administration form for clinical application.

\section{Financial \& competing interests disclosure}

This work is funded by the ERC Horizon 2020 project ERC-2014-StG - 637830. The authors have no other relevant affiliations or financial involvement with any organization or entity with a financial interest in or financial conflict with the subject matter or materials discussed in the manuscript apart from those disclosed.

No writing assistance was utilized in the production of this manuscript.

\section{Acknowledgments}

The authors would like to thank F Adams and G Costabile for diligent review of this manuscript.

\section{References}

1. Adams D, Gonzalez-Duarte A, O'riordan WD et al. Patisiran, an RNAi therapeutic, for hereditary transthyretin amyloidosis. N. Engl. J. Med. 379(1), 11-21 (2018).

2. Ruigrok MJR, Frijlink HW, Hinrichs WLJ. Pulmonary administration of small interfering RNA: the route to go? J. Control. Rel. 235, 14-23 (2016).

3. Thanki K, Blum KG, Thakur A, Rose F, Foged C. Formulation of RNA interference-based drugs for pulmonary delivery: challenges and opportunities. Ther. Deliv. 9(10), 731-749 (2018).

4. Aigner A, Kögel D. Nanoparticle/siRNA-based therapy strategies in glioma: which nanoparticles, which siRNAs? Nanomedicine (Lond.) 13(1), 89-103 (2018).

5. Weber S, Zimmer A, Pardeike J. Solid lipid nanoparticles (SLN) and nanostructured lipid carriers (NLC) for pulmonary application: a review of the state of the art. Eur. J. Pharm. Biopharm. 86(1), 7-22 (2014).

6. Levy ML, Hardwell A, Mcknight E, Holmes J. Asthma patients' inability to use a pressurised metered-dose inhaler (pMDI) correctly correlates with poor asthma control as defined by the global initiative for asthma (GINA) strategy: a retrospective analysis. Prim. Care Respir. J. 22(4), 406 (2013).

7. Saleem I, Diez F, Jones B, Kayali N, Polo L. Investigation on the aerosol performance of dry powder inhalation hypromellose capsules with different lubricant levels. Int. J. Pharm. 492(1-2), 258-263 (2015).

8. Demoly P, Hagedoorn P, De Boer AH, Frijlink HW. The clinical relevance of dry powder inhaler performance for drug delivery. Respir. Med. 108(8), 1195-1203 (2014).

9. Patil J, Sarasija S. Pulmonary drug delivery strategies: a concise, systematic review. Lung India 29(1), 44 (2012).

10. Yang MY, Chan JGY, Chan H-K. Pulmonary drug delivery by powder aerosols. J. Control. Rel. 193, 228-240 (2014).

11. Barham AS, Tewes F, Healy AM. Moisture diffusion and permeability characteristics of hydroxypropylmethylcellulose and hard gelatin capsules. Int. J. Pharm. 478(2), 796-803 (2015).

12. Liang W, Chan AY, Chow MY et al. Spray freeze drying of small nucleic acids as inhaled powder for pulmonary delivery. Asian J. Pharm. 13(2), 163-172 (2018).

13. Leng D, Thanki K, Foged C, Yang M. Formulating inhalable dry powders using two-fluid and three-fluid nozzle spray drying. Pharm. Res. 35(12), 247 (2018).

14. Liang W, Chow MY, Chow SF, Chan H-K, Kwok PC, Lam JK. Using two-fluid nozzle for spray freeze drying to produce porous powder formulation of naked siRNA for inhalation. Int. J. Pharm. 552(1-2), 67-75 (2018).

15. Okuda T, Morishita M, Mizutani K, Shibayama A, Okazaki M, Okamoto H. Development of spray-freeze-dried siRNA/PEI powder for inhalation with high aerosol performance and strong pulmonary gene silencing activity. J. Control. Rel. 279, 99-113 (2018).

16. Merkel OM, Rubinstein I, Kissel T. siRNA delivery to the lung: what's new? Adv. Drug Deliv. Rev. 75, 112-128 (2014).

17. Hoppentocht M, Hagedoorn P, Frijlink H, De Boer A. Technological and practical challenges of dry powder inhalers and formulations. Adv. Drug Deliv. Rev. 75, 18-31 (2014).

18. Ziaee A, Albadarin AB, Padrela L, Femmer T, O’reilly E, Walker G. Spray drying of pharmaceuticals and biopharmaceuticals: critical parameters and experimental process optimization approaches. Eur. J. Pharm. Sci. 127, 300-318 (2018).

19. Vehring R. Pharmaceutical particle engineering via spray drying. Pharm. Res. 25(5), 999-1022 (2008).

20. Chow MYT, Qiu Y, Lo FFK et al. Inhaled powder formulation of naked siRNA using spray drying technology with L-leucine as dispersion enhancer. Int. J. Pharm.530(1-2), 40-52 (2017). 
